\title{
Acute and Sub-acute Toxicity Study of Aqueous Extracts of Enicostemma axillare (Lam.) Raynal in Animal models
}

\author{
Aiyalu Rajasekaran* and Ramasamy Arivukkarasu \\ Department of Pharmaceutical Analysis, KMCH College of Pharmacy, Coimbatore, Tamil Nadu, India.
}

\begin{abstract}
Background: Enicostemma axillare (Lam.) Raynal is used in traditional practice for the treatment of diabetes, malaria and liver disorders. No systematic toxicity study was described for this plant and hence the present was undertaken to evaluate acute and sub-acute toxicity of aqueous extract of Enicostemma axillare (AEEA). Objective: The acute oral toxicity study of AEEA was carried out as per the OECD guidelines 423 in mice and the sub-acute toxicity was carried out as per the guidelines set by OECD 407 in male and female rats. Materials and Methods: Body weight, food and water consumption, hematological parameters, biochemical parameters, organ weight and histopathological analysis were carried out. Results: No gross toxicity and mortality was observed upto a dose of $2000 \mathrm{mg} / \mathrm{kg}$. For sub-acute toxicity test, $200 \mathrm{mg} / \mathrm{kg}$ and 400 $\mathrm{mg} / \mathrm{kg}$ daily dose of AEEA administered orally for 28 days in male and female group of rats not exhibited any signs of toxicity and mortality.
\end{abstract}

Conclusion: In acute oral toxicity study, the oral administration of AEEA in mice was found to be safe up to a dose of $2000 \mathrm{mg} / \mathrm{kg}$. Both male and female treated rats showed no change in hematological, biochemical and histological investigations and no signs of toxicity were observed upto the dose of $400 \mathrm{mg} / \mathrm{kg}$ in rats.

Key words: Acute toxicity, Enicostemma axillare, Histology, Sub-acute toxicity, OECD guidelines.

Corresponding author: Dr. Aiyalu Rajasekaran, Department of Pharmaceutical Analysis, KMCH College of Pharmacy Kovai Estate, Kalapatti Road, Coimbatore-641048. Tamilnadu, India.

Phone no: 09840132004, Fax no: +91-422-2369302

Email: rsekaran2001in@yahoo.co.in

DOI : 10.5530/pj.2016.1.3

\section{INTRODUCTION}

Enicostemma axillare (Lam.) Raynal (EA) also called as Vellarugu in Tamil and Chotachirayata in Hindi is an important group of medicinal plant belonging to the Gentianaceae family. EA (Figure 1) is a glabrous perennial rainy season herb, growing on moist, damp and shady ridges and slopes of the borders of cultivated fields, chiefly near the sea and often on black cotton soil. ${ }^{1}$ Whole plant of EA was reported for the presence of phenols, tannins, flavonoids, glycosides, anthraquinones and sterols. ${ }^{2}$ The decoctions of the leaves of EA are used in rheumatism, abdominal ulcers, diabetes, cold swelling, antipyretic, and cough and for stomachache. ${ }^{3}$ Anti-diabetic effect of the aqueous extract of EA on streptozotocin induced diabetes mellitus in rats was reported..$^{4-6}$ Antidiabetic efficacy on alloxan induced diabetes mellitus in rats for the extracts of EA was reported. ${ }^{7-10}$ Swertiamarin was isolated from $\mathrm{EA}^{11}$ and screened for antioxidant and hepatoprotective effect of compound against D-galactosamine induced acute liver damage in rats. Hepatoprotective effect of EA was reported on carbon tetrachloride induced hepatic damage in rats. ${ }^{12-13}$ Hypolipidaemic and antioxidant effect of aqueous extract of EA was reported in cholesterol fed rats. ${ }^{14}$ Aerial parts of EA was reported to possess hypolipidaemic effect in p-dimethyl aminobenzene (PDAB) induced hepatotoxic animals. ${ }^{15}$

In India, Enicostemma axillare is used by the traditional healers for the treatment of liver disorders, which we have scientifically validated in our earlier study in rats. ${ }^{16}$ To ascertain and establish the safety for its use in traditional practice, acute and sub-acute toxicity studies of the aqueous extract of Enicostemma axillare were carried out as per OCED guidelines.

\section{MATERIALS AND METHODS}

\section{Plant materials}

Enicostemma axillare was collected during August 2014 and the plants were authenticated (BSI/SRC/5/23/2014-15/Tech/873) by Dr. M. Palanisamy, Scientist C, Botanical survey of India, Coimbatore, India. Voucher specimens of the plants were deposited at the Department of Pharmacognosy, KMCH College of Pharmacy, and Coimbatore for future reference.

\section{Preparation of extracts}

Whole plant of Enicostemma axillare was cleaned with distilled water to remove earthy matter and residual materials. It was then shade dried at room temperature $\left(32 \pm 2^{\circ} \mathrm{C}\right)$ for 10 days, pulverized to coarse powder, passed through a \#40 mesh sieve. Then decoction was prepared by boiling $100 \mathrm{~g}$ of the ground plant material in $500 \mathrm{~mL}$ of distilled water for about $30 \mathrm{~min}$. Filtered and concentrated separately under reduced pressure IKA Rotary evaporator (Model No RN 10 digital V, ILMAC Germany) at $40^{\circ} \mathrm{C}$. Percentage yield of AEEA was $16.2 \% \mathrm{w} / \mathrm{w}$.

\section{Animals}

Female Albino mice (7-8 weeks old, 20-30 g) and Wistar strains of either sex rats (10-12 weeks old, 150-200 g) were obtained from the animal house of Kovai medical center research and educational trust, Coimbatore, India. The animals were kept in polypropylene cages at a temperature of $25 \pm 2{ }^{\circ} \mathrm{C}, \mathrm{RH}(50 \pm 5 \%), 12 \mathrm{~h}$ light and dark cycles. They were fed with standard laboratory animal diet and water ad libitum. Animals were acclimatized to laboratory conditions before the test. Experiments were designed and conducted in accordance with ethical norms approved by Committee for the Purpose of Control and Supervision on Experiments on Animals (CPCSEA) and Institutional Animal Ethical Committee (IAEC)(KMCRET/AICTE/01/2014-15, dt.31/01/2015).

\section{Acute toxicity study}

Acute toxicity test was performed according to $\mathrm{OECD}^{17}$ guideline 423 for testing of chemicals (2001). Healthy young adult albino nulliparous, nonpregnant female mice weighing about 20-30 g were administered as a single dose ( $1 \mathrm{ml}$ ) orally using oral feeding needle with 5, 50, 300 and 2000 $\mathrm{mg} / \mathrm{kg}$ of AEEA in distilled water. Animals were observed individually for first $30 \mathrm{~min}$, periodically during the first $24 \mathrm{~h}$, with special attention given during the first $4 \mathrm{~h}$, and daily thereafter, for a total of 14 days to observe toxicity signs like changes in skin and fur, eyes and mucous membranes, respiratory, circulatory, autonomic and central nervous systems and behavioural pattern. On $15^{\text {th }}$ day animals were anaesthetized, blood was 


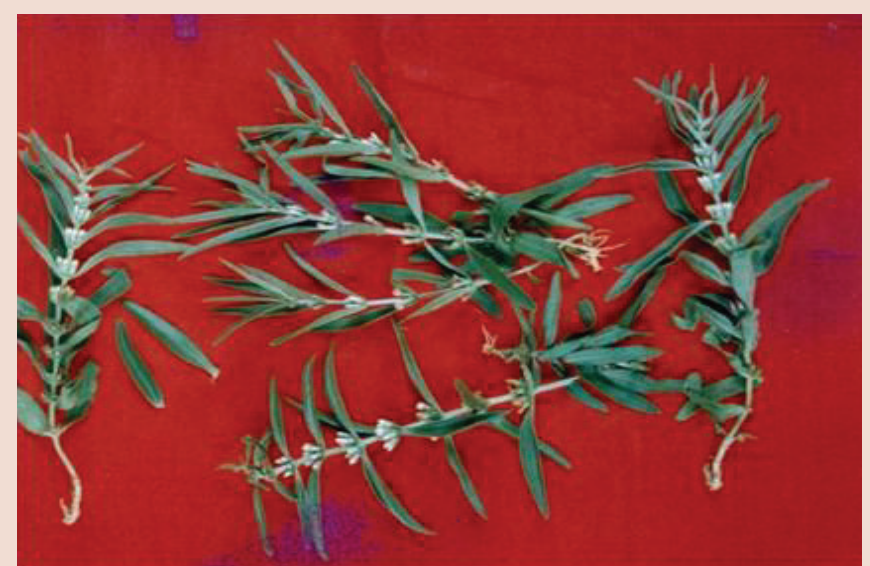

Figure 1: Entire plant of Enicostemma axillare

collected from the animals for haematological and biochemical analysis. Mice were then sacrificed, dissected and the organs lungs, liver, spleen and kidney were carefully collected, weighed, processed and observed under photomicroscope for histopathological examination.

\section{Sub-acute toxicity study}

The sub-acute toxicity assessment of AEEA was performed as per OECD Guideline $407 .{ }^{18}$ Wistar strain of rats (150-200 g) was divided into six groups, each consisting of six male and six female rats. Male and female Control groups received only distilled water. Two other groups of each male and female were administered with AEEA in distilled water orally using rat oral feeding needles, daily for 28 days at a dose of $200 \mathrm{mg} / \mathrm{kg}$ and $400 \mathrm{mg} / \mathrm{kg}$ respectively. During the treatment period the body weight of animals were monitored on $0,7^{\text {th }}, 14^{\text {th }}, 21^{\text {st }}$ and $28^{\text {th }}$ day. Food consumption and water intake for all the groups were observed from day 1 to 28 days.

On $29^{\text {th }}$ day, the overnight fasted rats were anaesthetized with diethyl ether inhalation in a jar containing cotton soaked with diethyl ether. Then blood samples were withdrawn from retro-orbital sinus and the collected blood samples were evaluated for hematological parameters viz. Red blood cells (RBC), White blood cells (WBC), Hemoglobin (Hb), Platelet count, Packed cell volume (PCV), Differential count, Mean Platelet Volume (MPV), Mean corpuscular volume (MCV), Mean corpuscular hemoglobin $(\mathrm{MCH})$, Mean corpuscular hemoglobin concentration (MCHC) and Red blood cell distribution width (RDW).

A portion of the blood samples were centrifuged at $10000 \mathrm{rpm}$ for $10 \mathrm{~min}$ and the separated serum was analyzed for biochemical parameters viz. Cholesterol, triglycerides, VLDL levels SGOT, SGPT, ALP, total bilirubin, total protein, albumin, globulin, urea, uric acid and creatinine. Biochemical investigations were carried out in an auto analyser (Photometer 5010 V5+, Robert Riely, Berlin) using Piramal healthcare limited reagent kit. The animals were sacrificed by cervical dislocation and the organs, heart, liver, spleen, kidneys, stomach, testes and ovary were isolated and weighed processed and observed under photomicroscope for histopathological examination.

\section{Statistical analysis}

The results are expressed as the mean \pm SEM. The significance of the difference was evaluated by one-way ANOVA followed by Dunnett's test. Data were considered statistically significant if $\mathrm{p}<0.05$.

\section{RESULTS}

\section{Acute Toxicity Study}

Single oral administration of AEEA at a dose of 5, 50, 300 and $2000 \mathrm{mg} /$ $\mathrm{kg}$ as per OECD guideline 423 for 14 days did not produce any mortality in tested animals (Table 1). No observable sign of toxicity was detected during the experimental period.

\section{Sub-acute toxicity study}

Daily oral administration of AEEA at a dose of $200 \mathrm{mg} / \mathrm{kg}$ and $400 \mathrm{mg} / \mathrm{kg}$ elicited no change in skin and fur, eyes and mucous membranes, and also respiratory, circulatory, autonomic and central nervous systems and behaviour pattern of the animals observed. Absence of tremors, convulsions, salivation, diarrhoea, and sleep also noted (Table 2). Thus in sub-acute toxicity study,

\section{Table 1: Observations of Acute toxicity study of AEEA}

\begin{tabular}{cccccc}
\hline Group & $\begin{array}{c}\text { Dose } \\
(\mathbf{m g} / \mathbf{k g})\end{array}$ & $\begin{array}{c}\text { Number of } \\
\text { animals }\end{array}$ & $\begin{array}{c}\text { Number } \\
\text { of survival }\end{array}$ & $\begin{array}{c}\text { Number } \\
\text { of deaths }\end{array}$ & $\begin{array}{c}\text { Percentage } \\
\text { of mortality }\end{array}$ \\
\hline 1 & Control & 3 & 3 & 0 & 0 \\
2 & 5 & 3 & 3 & 0 & 0 \\
3 & 50 & 3 & 3 & 0 & 0 \\
4 & 300 & 3 & 3 & 0 & 0 \\
5 & 2000 & 3 & 3 & 0 & 0 \\
\hline
\end{tabular}

Table 2: Behavioural Studies of AEEA at a dose 200 and $400 \mathrm{mg} / \mathrm{kg}$ on rats in sub-acute toxicity study

\begin{tabular}{|c|c|c|c|c|c|c|c|c|c|c|c|c|c|c|c|c|c|c|c|}
\hline Gross activity & $\begin{array}{c}30 \\
\text { Min }\end{array}$ & $1 h$ & $2 \mathrm{~h}$ & $3 \mathbf{h}$ & $4 \mathrm{~h}$ & $24 h$ & $\begin{array}{c}\text { Day } \\
3\end{array}$ & $\begin{array}{c}\text { Day } \\
5\end{array}$ & $\begin{array}{c}\text { Day } \\
7\end{array}$ & $\begin{array}{c}\text { Day } \\
9\end{array}$ & $\begin{array}{c}\text { Day } \\
11\end{array}$ & $\begin{array}{c}\text { Day } \\
13\end{array}$ & $\begin{array}{c}\text { Day } \\
15\end{array}$ & $\begin{array}{c}\text { Day } \\
17\end{array}$ & $\begin{array}{c}\text { Day } \\
19\end{array}$ & $\begin{array}{c}\text { Day } \\
21\end{array}$ & $\begin{array}{c}\text { Day } \\
23\end{array}$ & $\begin{array}{c}\text { Day } \\
25\end{array}$ & $\begin{array}{c}\text { Day } \\
28\end{array}$ \\
\hline Respiration & + & + & + & + & + & + & + & + & + & + & + & + & + & + & + & + & + & + & + \\
\hline Writhing & - & - & - & - & - & - & - & - & - & - & - & - & - & - & - & - & - & - & 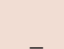 \\
\hline Tremor & - & - & - & - & - & - & - & - & - & - & - & - & - & - & - & - & - & - & - \\
\hline Convulsion & - & - & - & - & - & - & - & - & - & - & - & - & - & - & - & - & - & - & - \\
\hline Sense of touch/sound & + & + & + & + & + & + & + & + & + & + & + & + & + & + & + & + & + & + & + \\
\hline Salivation & + & + & + & + & + & + & + & + & + & + & + & + & + & + & + & + & + & + & + \\
\hline Urination & + & + & + & + & + & + & + & + & + & + & + & + & + & + & + & + & + & + & + \\
\hline Defecation & + & + & + & + & + & + & + & + & + & + & + & + & + & + & + & + & + & + & + \\
\hline Diarrhoea & - & - & - & - & - & - & - & - & - & - & - & - & - & - & - & - & - & - & - \\
\hline Sedation & - & - & - & - & - & - & - & - & - & - & - & - & - & - & - & - & - & - & - \\
\hline Locomotor & + & + & + & + & + & + & + & + & + & + & + & + & + & + & + & + & + & + & + \\
\hline edema & - & - & - & - & - & - & - & - & - & - & - & - & - & - & - & - & - & - & - \\
\hline Mortality & - & - & - & - & - & - & - & - & - & - & - & - & - & - & - & - & - & - & - \\
\hline
\end{tabular}

+ Indicates normal; - Indicates no effect. 
no sign of toxicity viz. jerk, convulsion and mortality was observed for both the male and female rats treated with 200 and $400 \mathrm{mg} / \mathrm{kg}$ dose of AEEA.

\section{Parameters observed during acute and sub-acute toxicity studies}

Body weight

Gain in body weight of mice and rats was observed in acute and sub-acute toxicity study respectively for control group and AEEA administered animals and the results are recorded in Table 3 and 4.
Table 3: Assessment of body weights of mice in acute toxicity study

\begin{tabular}{ccccc}
\hline \multicolumn{4}{c}{ Body weight $(\mathrm{g})$} \\
Treatment & 0 day & $7^{\text {th }}$ day & $14^{\text {th }}$ day & $\begin{array}{c}\text { Weight gain } \\
\text { on } 14^{\text {th }} \text { day }\end{array}$ \\
\hline $\begin{array}{c}\text { Control } \\
\text { AEEA 2000 } \\
\text { mg } / \mathrm{kg}\end{array}$ & $19.98 \pm 0.52$ & $21.75 \pm 0.35$ & $23.91 \pm 0.49$ & $3.93 \pm 0.36$ \\
\hline
\end{tabular}

Data provided as mean \pm SEM $(\mathrm{n}=6) ;{ }^{\mathrm{a}} \mathrm{p}<0.05$ treated groups vs control ${ }^{b} \mathrm{p}<0.01$ treated groups Vs control; $\mathrm{p}<<0.001$ treated groups vs control.

Table 4: Assessment of body weights of rats in sub-acute toxicity study

\begin{tabular}{|c|c|c|c|c|c|c|}
\hline Groups & Day 1 & Day 7 & Day 14 & Day 21 & Day 28 & $\begin{array}{l}\text { Weight gain (g) } \\
\text { on day } 28 \text { th day }\end{array}$ \\
\hline Male Control & $153.6 \pm 1.49$ & $182.16 \pm 2.61$ & $212.66 \pm 2.94$ & $231.66 \pm 4.58$ & $250.5 \pm 1.62$ & $96.83 \pm 2.72$ \\
\hline $\begin{array}{l}\text { Male AEEA } \\
200 \mathrm{mg} / \mathrm{kg}\end{array}$ & $127.66 \pm 1.35$ & $144.5 \pm 2.89$ & $163.66 \pm 2.99$ & $184.5 \pm 3.25$ & $200.16 \pm 4.06$ & $71.33 \pm 3.06 b$ \\
\hline $\begin{array}{l}\text { Male AEEA } \\
400 \mathrm{mg} / \mathrm{kg}\end{array}$ & $167.33 \pm 1.94$ & $190.33 \pm 5.55$ & $199.16 \pm 4.74$ & $229.0 \pm 2.46$ & $247.5 \pm 2.54$ & $80.16 \pm 2.79 b$ \\
\hline Female Control & $124.66 \pm 0.98$ & $146.3 \pm 0.71$ & $164.33 \pm 1.66$ & $182.0 \pm 2.35$ & $199.5 \pm 2.37$ & $74.83 \pm 2.45$ \\
\hline $\begin{array}{l}\text { Female AEEA } \\
200 \mathrm{mg} / \mathrm{kg}\end{array}$ & $132.5 \pm 1.76$ & $151.0 \pm 4.179$ & $168.66 \pm 5.53$ & $187.66 \pm 6.23$ & $205.66 \pm 3.52$ & $73.16 \pm 4.30 \mathrm{c}$ \\
\hline $\begin{array}{c}\text { Female AEEA } \\
400 \mathrm{mg} / \mathrm{kg}\end{array}$ & $154.16 \pm 1.49$ & $182.5 \pm 1.05$ & $199.16 \pm 2.72$ & $217.66 \pm 3.49$ & $233.66 \pm 4.01$ & $81.16 \pm 5.54 c$ \\
\hline
\end{tabular}

Data provided as mean $\pm \operatorname{SEM}(\mathrm{n}=6)$; ap $<0.05$ treated groups vs control

$\mathrm{bp}<0.01$ treated groups Vs control; $\mathrm{cp}<0.001$ treated groups vs control.

\section{Food and distilled water consumption}

Food and distilled water consumption of mice (acute toxicity) and rats (sub-acute toxicity) were continuously monitored, where there is no change in consumption was observed for treated groups and the control group animals ( Figure 2 to Figure 5).

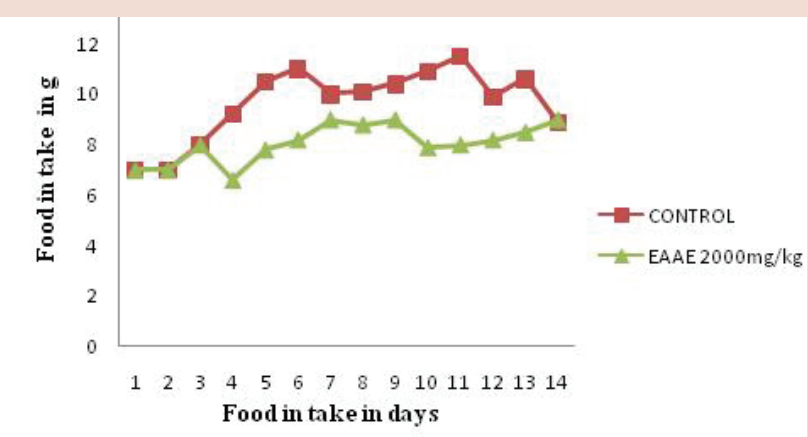

Figure 2: Food intake in g during acute toxicity study for 14 days

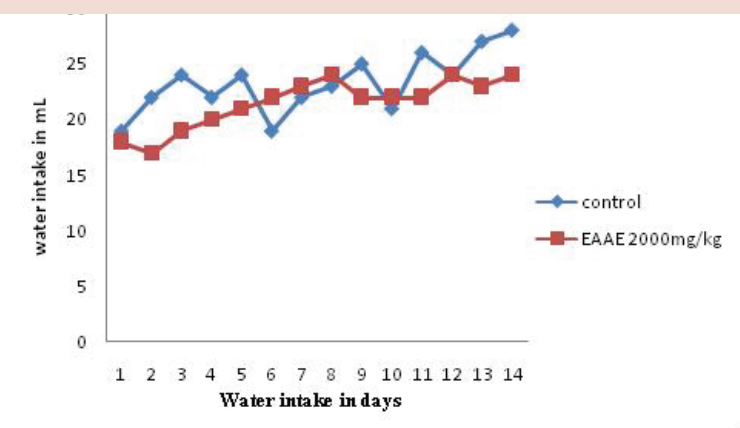

Figure 3: Water intake in $\mathrm{ml}$ during acute toxicity study for 14 days
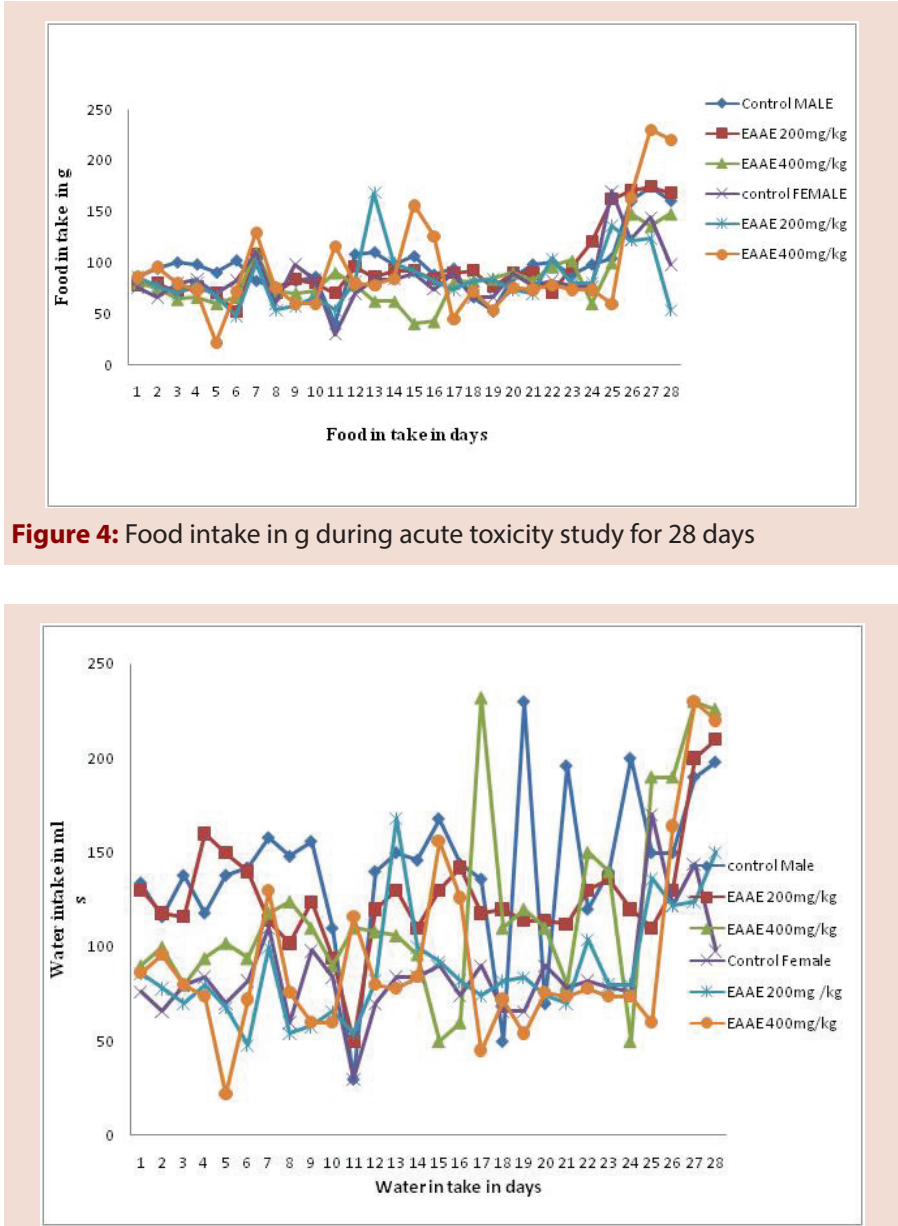

Figure 5: Water intake in $\mathrm{mL}$ during acute toxicity study for 28 days 


\section{Hematological analysis}

The results of hematological investigations (Table 5 and 6) conducted on day $14^{\text {th }}$ day for acute toxicity study and on $29^{\text {th }}$ day for sub-acute toxicity study revealed no significant changes in the values of $\mathrm{RBC}, \mathrm{WBC}, \mathrm{Hb}$, platelet count, $\mathrm{PCV}$, differential count, $\mathrm{MPV}, \mathrm{MCV}, \mathrm{MCH}, \mathrm{MCHC}$ and $\mathrm{RDW}$ of treated groups when compared with the respective control mice and rats respectively.

\section{Biochemical Investigations}

Biochemical investigations were performed in order to review any toxic effects produced after administration of AEEA on liver and kidney. There was no significant alteration in cholesterol triglycerides and VLDL levels in treated groups of sub-acute toxicity study when compared with control group of rats (Table 7). No significant change observed in (serum glutamic oxaloacetic transaminase) SGOT, (Serum glutamic pyruvic transaminase) SGPT, (alkaline phosphatase) ALP and total bilirubin content of treated group animals when compared with control group animals (Table 8 and 9). There was no significant alteration observed in creatinine, urea and uric acid levels of treated group animals when compared with control group animals (Table 10 and 11).

Table 5: Evaluation of hematological parameters of mice in acute toxicity study

\begin{tabular}{|c|c|c|c|c|c|c|c|c|c|c|c|c|c|c|}
\hline \multirow{2}{*}{ Groups } & \multirow{2}{*}{$\begin{array}{c}\text { Total RBC } \\
\left(\times 10^{6} \text { cells } /\right. \\
\left.\mathrm{mm}^{3}\right)\end{array}$} & \multirow{2}{*}{$\begin{array}{c}\text { Total Hb } \\
\text { (g/dl) }\end{array}$} & \multirow{2}{*}{$\begin{array}{c}\text { Total WBC } \\
\left(\times 10^{6} \mathrm{cells} /\right. \\
\left.\mathrm{mm}^{3}\right)\end{array}$} & \multirow{2}{*}{$\begin{array}{c}\text { Platelet } \\
\text { Count } \\
\left(\mathrm{X} 10^{6} \mathrm{cells} /\right. \\
\left.\mathrm{mm}^{3}\right)\end{array}$} & \multirow{2}{*}{$\begin{array}{l}\text { PCV } \\
\text { (\%) }\end{array}$} & \multicolumn{4}{|c|}{ Differential Count } & \multirow{2}{*}{$\begin{array}{c}\text { MPV } \\
\text { (fL) }\end{array}$} & \multirow{2}{*}{$\begin{array}{c}\text { MCV } \\
\mathbf{f L})\end{array}$} & \multirow{2}{*}{$\begin{array}{c}\text { MCH } \\
\text { (pg) }\end{array}$} & \multirow{2}{*}{$\begin{array}{c}\mathrm{MCHC}(\mathrm{g} / \\
\mathrm{dL})\end{array}$} & \multirow{2}{*}{ RDW (\%) } \\
\hline & & & & & & $\begin{array}{c}\text { Polymorphs } \\
\text { (\%) }\end{array}$ & $\begin{array}{c}\text { Lymphocytes } \\
\text { (\%) }\end{array}$ & $\begin{array}{c}\text { Monocytes } \\
\text { (\%) }\end{array}$ & $\begin{array}{c}\text { Eosnophils } \\
(\%)\end{array}$ & & & & & \\
\hline Control & $\begin{array}{c}14.40 \pm \\
0.69\end{array}$ & $\begin{array}{c}14.48 \pm \\
0.42\end{array}$ & $\begin{array}{c}13.98 \pm \\
0.75\end{array}$ & $\begin{array}{c}789.5 \pm \\
13.67\end{array}$ & $\begin{array}{c}45.58 \pm \\
1.49\end{array}$ & $5.16 \pm 0.30$ & $80.50 \pm 2.23$ & $3.66 \pm 0.42$ & $2.83 \pm 0.30$ & $\begin{array}{c}8.05 \pm \\
0.31\end{array}$ & $\begin{array}{c}60.3 \pm \\
0.43\end{array}$ & $\begin{array}{c}19.15 \pm \\
0.19\end{array}$ & $\begin{array}{c}32.31 \pm \\
0.54\end{array}$ & $18.9 \pm 0.48$ \\
\hline $\begin{array}{c}\text { AEEA } 2000 \\
\mathrm{mg} / \mathrm{kg}\end{array}$ & $\begin{array}{c}16.25 \pm \\
0.73^{\mathrm{a}}\end{array}$ & $\begin{array}{c}13.82 \pm \\
0.73^{\mathrm{a}}\end{array}$ & $\begin{array}{c}13.55 \pm \\
0.57^{\mathrm{a}}\end{array}$ & $\begin{array}{c}757.16 \pm \\
32.89^{\mathrm{a}}\end{array}$ & $\begin{array}{c}42.33 \pm \\
0.63\end{array}$ & $4.83 \pm 0.60^{\mathrm{a}}$ & $85.33 \pm 2.41^{\mathrm{a}}$ & $3.0 \pm 0.36^{\mathrm{a}}$ & $3.66 \pm 0.55^{a}$ & $\begin{array}{c}8.01 \pm \\
0.27^{\mathrm{a}}\end{array}$ & $\begin{array}{c}60.01 \pm \\
1.02^{\mathrm{a}}\end{array}$ & $\begin{array}{c}19.15 \pm \\
0.18^{\mathrm{a}}\end{array}$ & $\begin{array}{c}32.06 \pm \\
0.55^{\mathrm{a}}\end{array}$ & $\begin{array}{c}17.66 \pm \\
0.79^{\mathrm{a}}\end{array}$ \\
\hline
\end{tabular}

Data provided as mean \pm SEM $(n=6)$; ap $>0.05$ treated groups vs control.

\section{Table 6: Evaluation of hematological parameters of rats in sub-acute toxicity study}

\begin{tabular}{|c|c|c|c|c|c|c|c|c|c|c|c|c|c|c|}
\hline Groups & $\begin{array}{c}\text { Total RBC } \\
\left(\mathrm{X} 10^{6}\right. \\
\left.\text { cells } / \mathrm{mm}^{3}\right)\end{array}$ & $\begin{array}{l}\text { Total Hb } \\
\text { (g/dl) }\end{array}$ & $\begin{array}{c}\text { Total WBC } \\
\left(\mathrm{X} 10^{6}\right. \\
\left.\text { cells } / \mathrm{mm}^{3}\right)\end{array}$ & $\begin{array}{c}\text { Platelet } \\
\text { Count }\left(X 10^{6}\right. \\
\left.\text { cells } / \mathrm{m}^{3}\right)\end{array}$ & PCV (\%) & $\begin{array}{c}\text { Polymorphs } \\
\text { (\%) }\end{array}$ & $\begin{array}{l}\text { Differentia } \\
\text { Lymphocytes } \\
\text { (\%) }\end{array}$ & $\begin{array}{c}\text { Monocytes } \\
\text { (\%) }\end{array}$ & $\begin{array}{c}\text { Eosnophils } \\
(\%)\end{array}$ & $\begin{array}{c}\text { MPV } \\
\text { (fL) }\end{array}$ & MCV fL) & $\begin{array}{r}\text { MCH } \\
(\mathrm{pg})\end{array}$ & $\begin{array}{l}\text { MCHC } \\
\text { (g/dL) }\end{array}$ & $\begin{array}{l}\text { RDW } \\
(\%)\end{array}$ \\
\hline $\begin{array}{l}\text { Control } \\
\text { Male }\end{array}$ & $\begin{array}{c}4.98 \pm \\
0.13\end{array}$ & $\begin{array}{c}14.95 \pm \\
0.590\end{array}$ & $\begin{array}{c}11.55 \pm \\
0.82\end{array}$ & $\begin{array}{c}740.16 \pm \\
40.67\end{array}$ & $\begin{array}{c}46.48 \pm \\
1.66\end{array}$ & $5.0 \pm 1.06$ & $84.83 \pm 2.05$ & $3.33 \pm 0.21$ & $\begin{array}{c}5.66 \pm \\
0.55\end{array}$ & $\begin{array}{c}7.33 \pm \\
0.20\end{array}$ & $\begin{array}{c}63.95 \pm \\
0.73\end{array}$ & $\begin{array}{c}22.25 \pm \\
0.68\end{array}$ & $\begin{array}{c}31.88 \pm \\
0.35\end{array}$ & $\begin{array}{c}17.84 \pm \\
0.41\end{array}$ \\
\hline $\begin{array}{c}\text { Male } \\
\text { AEEA } 200 \\
\text { mg/kg }\end{array}$ & $\begin{array}{l}4.94 \pm \\
0.46^{\mathrm{a}}\end{array}$ & $\begin{array}{c}15.31 \pm \\
1.19^{\mathrm{a}}\end{array}$ & $\begin{array}{c}11.7 \pm \\
1.25^{\mathrm{a}}\end{array}$ & $\begin{array}{c}808.33 \pm \\
23.93^{\mathrm{a}}\end{array}$ & $\begin{array}{l}45.8 \pm \\
3.39^{\mathrm{a}}\end{array}$ & $6.83 \pm 1.60^{\mathrm{a}}$ & $84.16 \pm 2.24^{\mathrm{a}}$ & $3.5 \pm 0.71^{\mathrm{a}}$ & $\begin{array}{c}3.83 \pm \\
0.40^{\mathrm{a}}\end{array}$ & $\begin{array}{c}7.96 \pm \\
0.22^{\mathrm{a}}\end{array}$ & $\begin{array}{c}66.86 \pm \\
1.57^{\mathrm{a}}\end{array}$ & $\begin{array}{c}22.58 \pm \\
0.61^{\mathrm{a}}\end{array}$ & $\begin{array}{c}28.33 \pm \\
2.37^{\mathrm{a}}\end{array}$ & $\begin{array}{c}17.11 \pm \\
0.45^{\mathrm{a}}\end{array}$ \\
\hline $\begin{array}{c}\text { Male } \\
\text { AEEA } 400 \\
\mathrm{mg} / \mathrm{kg}\end{array}$ & $\begin{array}{c}4.58 \pm \\
0.18^{\mathrm{a}}\end{array}$ & $\begin{array}{c}14.66 \pm \\
0.73^{\mathrm{a}}\end{array}$ & $\begin{array}{c}12.13 \pm \\
0.90^{\mathrm{a}}\end{array}$ & $\begin{array}{c}735.66 \pm \\
38.92^{\mathrm{a}}\end{array}$ & $\begin{array}{c}42.86 \pm \\
1.83^{\mathrm{a}}\end{array}$ & $4.83 \pm 1.07^{a}$ & $86.0 \pm 2.23^{a}$ & $3.66 \pm 0.33^{a}$ & $\begin{array}{l}5.0 \pm \\
0.77^{\mathrm{a}}\end{array}$ & $\begin{array}{c}7.431 \pm \\
0.23^{\mathrm{a}}\end{array}$ & $\begin{array}{c}65.36 \pm \\
0.50^{\mathrm{a}}\end{array}$ & $\begin{array}{c}21.26 \pm \\
0.37^{\mathrm{a}}\end{array}$ & $\begin{array}{c}31.85 \pm \\
0.77^{\mathrm{a}}\end{array}$ & $\begin{array}{c}17.42 \pm \\
0.32^{\mathrm{a}}\end{array}$ \\
\hline $\begin{array}{l}\text { Control } \\
\text { Female }\end{array}$ & $\begin{array}{c}5.0 \pm \\
0.10\end{array}$ & $\begin{array}{c}15.36 \pm \\
0.36\end{array}$ & $\begin{array}{c}11.65 \pm \\
0.39\end{array}$ & $\begin{array}{c}777.83 \pm \\
30.75\end{array}$ & $\begin{array}{c}52.78 \pm \\
0.96\end{array}$ & $5.00 \pm 0.81$ & $86.16 \pm 1.60$ & $3.76 \pm 0.33$ & $\begin{array}{c}5.66 \pm \\
0.33\end{array}$ & $\begin{array}{l}8.1 \pm \\
0.06\end{array}$ & $\begin{array}{c}71.5 \pm \\
1.54\end{array}$ & $\begin{array}{c}32.88 \pm \\
0.36\end{array}$ & $\begin{array}{c}32.80 \pm \\
0.36\end{array}$ & $\begin{array}{c}18.45 \pm \\
0.14\end{array}$ \\
\hline $\begin{array}{c}\text { Female } \\
\text { AEEA } 200 \\
\text { mg/kg }\end{array}$ & $\begin{array}{c}5.66 \pm \\
0.40^{\mathrm{a}}\end{array}$ & $\begin{array}{c}16.61 \pm \\
0.20^{\mathrm{a}}\end{array}$ & $\begin{array}{c}12.28 \pm \\
0.91^{\mathrm{a}}\end{array}$ & $\begin{array}{c}823.83 \pm \\
12.65^{\mathrm{a}}\end{array}$ & $\begin{array}{c}54.36 \pm \\
1.31^{\mathrm{a}}\end{array}$ & $6.83 \pm 0.48^{a}$ & $90.33 \pm 2.49^{\mathrm{a}}$ & $4.33 \pm 0.42^{\mathrm{a}}$ & $\begin{array}{l}5.0 \pm \\
0.25^{\mathrm{a}}\end{array}$ & $\begin{array}{l}8.2 \pm \\
0.15^{\mathrm{a}}\end{array}$ & $\begin{array}{c}73.66 \pm \\
1.22^{\mathrm{a}}\end{array}$ & $\begin{array}{c}32.93 \pm \\
0.36^{\mathrm{a}}\end{array}$ & $\begin{array}{c}32.40 \pm \\
0.35^{\mathrm{a}}\end{array}$ & $\begin{array}{c}17.83 \pm \\
0.31^{\mathrm{a}}\end{array}$ \\
\hline $\begin{array}{c}\text { Female } \\
\text { AEEA } 400 \\
\text { mg/kg }\end{array}$ & $\begin{array}{c}4.83 \pm \\
0.31^{\mathrm{a}}\end{array}$ & $\begin{array}{c}14.81 \pm \\
0.31^{\mathrm{a}}\end{array}$ & $\begin{array}{c}12.54 \pm \\
0.69\end{array}$ & $\begin{array}{c}836.16 \pm \\
10.87^{\mathrm{a}}\end{array}$ & $\begin{array}{c}50.95 \pm \\
1.17^{\mathrm{a}}\end{array}$ & $5.83 \pm 0.30^{\mathrm{a}}$ & $89.00 \pm 1.15^{\mathrm{a}}$ & $4.50 \pm 0.34^{\mathrm{a}}$ & $\begin{array}{c}5.66 \pm \\
0.49^{a}\end{array}$ & $\begin{array}{c}8.41 \pm \\
0.13^{\mathrm{a}}\end{array}$ & $\begin{array}{c}70.91 \pm \\
0.58^{\mathrm{a}}\end{array}$ & $\begin{array}{c}33.5 \pm \\
0.46^{\mathrm{a}}\end{array}$ & $\begin{array}{c}34.28 \pm \\
0.35^{\mathrm{a}}\end{array}$ & $\begin{array}{c}18.48 \pm \\
0.14^{\mathrm{a}}\end{array}$ \\
\hline
\end{tabular}

Data provided as mean \pm SEM $(n=6) ;{ }^{\mathrm{a}} \mathrm{p}>0.05$ treated groups vs control.

Table 7: Effect of sub-acute dose of AEEA on lipid profile of rats

\begin{tabular}{|c|c|c|c|c|}
\hline Groups & $\begin{array}{l}\text { Dose mg/ } \\
\text { kg (p.o.) }\end{array}$ & $\begin{array}{l}\text { Cholesterol } \\
\text { (mg/dl) }\end{array}$ & $\begin{array}{l}\text { Triglycerides } \\
\text { (mg/dl) }\end{array}$ & $\begin{array}{l}\text { VLDL (mg/ } \\
\text { dl) }\end{array}$ \\
\hline Control Male & $\begin{array}{c}\text { Distilled } \\
\text { water }(5 \mathrm{ml} / \\
\mathrm{kg})\end{array}$ & $95.05 \pm 5.76$ & $262.43 \pm 34.98$ & $52.58 \pm 7.03$ \\
\hline Male AEEA & 200 mg/kg & $93.51 \pm 3.13^{\mathrm{a}}$ & $217.36 \pm 39.86^{\mathrm{a}}$ & $43.47 \pm 7.97^{a}$ \\
\hline Male & $400 \mathrm{mg} / \mathrm{kg}$ & $110.95 \pm 3.47^{\mathrm{a}}$ & $290.03 \pm 7.66^{a}$ & $58.00 \pm 1.53^{\mathrm{a}}$ \\
\hline $\begin{array}{l}\text { Control } \\
\text { Female }\end{array}$ & $\begin{array}{l}\text { Distilled } \\
\text { water } \\
(5 \mathrm{ml} / \mathrm{kg})\end{array}$ & $89.03 \pm 4.50$ & $295.76 \pm 14.55$ & $59.15 \pm 2.91$ \\
\hline Female AEEA & $200 \mathrm{mg} / \mathrm{kg}$ & $93.51 \pm 3.09^{\mathrm{a}}$ & $290.66 \pm 17.61^{a}$ & $58.13 \pm 3.52^{\mathrm{a}}$ \\
\hline Female AEEA & $400 \mathrm{mg} / \mathrm{kg}$ & $96.0 \pm 1.61^{\mathrm{a}}$ & $288.66 \pm 7.07^{\mathrm{a}}$ & $57.73 \pm 1.4^{\mathrm{a}}$ \\
\hline
\end{tabular}

Data provided as mean $\pm \operatorname{SEM}(n=6) ;{ }^{\mathrm{a}} \mathrm{p}>0.05$ treated groups vs control.
Table 8: Assessment of liver function test of mice in acute toxicity study

\begin{tabular}{cccccc}
\hline Groups & $\begin{array}{c}\text { Dose } \mathrm{mg} / \mathrm{kg} \\
(\boldsymbol{p} . \mathbf{o})\end{array}$ & $\begin{array}{c}\text { SGOT } \\
\text { (U/L) }\end{array}$ & $\begin{array}{c}\text { SGPT } \\
\text { (U/L) }\end{array}$ & $\begin{array}{c}\text { ALP } \\
\text { (U/L) }\end{array}$ & $\begin{array}{c}\text { Total } \\
\text { Bilirubin } \\
\text { (mg/dl) }\end{array}$ \\
\hline \multirow{2}{*}{ Control } & Distilled water & $33.01 \pm 1.01$ & $29.08 \pm$ & $249.08 \pm$ & $0.8 \pm$ \\
& $(5 \mathrm{ml} / \mathrm{kg})$ & 2.03 & 6.83 & 0.07 \\
AEEA & $2000 \mathrm{mg} / \mathrm{kg}$ & $37.08 \pm$ & $33.61 \pm$ & $251.23 \pm$ & $0.83 \pm 0.09^{\mathrm{a}}$ \\
& & $0.98^{\mathrm{a}}$ & $1.88^{\mathrm{a}}$ & $9.88^{\mathrm{a}}$ & \\
\hline
\end{tabular}

Data provided as mean \pm SEM $(n=6) ;{ }^{a} \mathrm{p}>0.05$ treated groups vs control.

\section{Organ weight}

No abnormal change in the relative weight of internal organs of mice and rats was observed when compared to control group as shown in Table 12 and 13. 


\section{Table 9: Assessment of liver function test of rats in sub-acute toxicity study}

\begin{tabular}{|c|c|c|c|c|c|c|c|c|}
\hline Groups & $\begin{array}{c}\text { Dose } \\
\mathrm{mg} / \mathrm{kg} \\
(\text { p.o. })\end{array}$ & $\begin{array}{l}\text { SGOT } \\
(\mathrm{U} / \mathrm{L})\end{array}$ & $\begin{array}{l}\text { SGPT } \\
\text { (U/L) }\end{array}$ & $\operatorname{ALP}(U / L)$ & $\begin{array}{c}\text { Total } \\
\text { ) Bilirubin } \\
\text { (mg/dl) }\end{array}$ & $\begin{array}{c}\text { Total } \\
\text { protein } \\
\text { (g/dl) }\end{array}$ & $\begin{array}{c}\text { Albumin } \\
\text { (g/dl) }\end{array}$ & $\begin{array}{c}\text { Globulin } \\
\text { (g/L) }\end{array}$ \\
\hline $\begin{array}{l}\text { Control } \\
\text { male }\end{array}$ & $\begin{array}{c}\text { Distilled } \\
\text { water } \\
(5 \mathrm{ml})\end{array}$ & $\begin{array}{c}54.29 \pm \\
4.38\end{array}$ & $\begin{array}{c}48.25 \pm \\
2.07\end{array}$ & $\begin{array}{c}237.35 \pm \\
16.92\end{array}$ & $\begin{array}{c}0.76 \pm \\
0.09\end{array}$ & $\begin{array}{c}7.23 \pm \\
0.28\end{array}$ & $\begin{array}{c}3.8 \pm \\
0.18\end{array}$ & $\begin{array}{c}3.08 \pm \\
0.29\end{array}$ \\
\hline $\begin{array}{c}\text { Male } \\
\text { AEEA }\end{array}$ & 200 & $\begin{array}{c}38.58 \pm \\
7.47^{\mathrm{a}}\end{array}$ & $\begin{array}{c}36.56 \pm \\
1.39^{\mathrm{a}}\end{array}$ & $\begin{array}{c}238.53 \pm \\
18.29^{\mathrm{a}}\end{array}$ & $\begin{array}{l}0.82 \pm \\
0.01^{\mathrm{a}}\end{array}$ & $\begin{array}{c}8.03 \pm \\
0.22^{\mathrm{a}}\end{array}$ & $\begin{array}{c}3.38 \pm \\
0.26^{\mathrm{a}}\end{array}$ & $\begin{array}{l}4.41 \pm \\
0.31^{\text {a }}\end{array}$ \\
\hline $\begin{array}{c}\text { Male } \\
\text { AEEA }\end{array}$ & 400 & $\begin{array}{c}57.36 \pm \\
5.20^{\mathrm{a}}\end{array}$ & $\begin{array}{c}48.95 \pm \\
6.23^{\mathrm{a}}\end{array}$ & $\begin{array}{c}231.21 \pm \\
31.22^{\mathrm{a}}\end{array}$ & $\begin{array}{l}0.95 \pm \\
0.05^{\mathrm{a}}\end{array}$ & $\begin{array}{c}7.75 \pm \\
0.31^{\mathrm{a}}\end{array}$ & $\begin{array}{l}3.8 \pm \\
0.18^{\mathrm{a}}\end{array}$ & $\begin{array}{c}3.76 \pm \\
0.35^{\text {a }}\end{array}$ \\
\hline $\begin{array}{l}\text { Control } \\
\text { Female }\end{array}$ & $\begin{array}{c}\text { Distilled } \\
\text { water } \\
(5 \mathrm{ml})\end{array}$ & $\begin{array}{c}51.33 \pm \\
1.85\end{array}$ & $\begin{array}{c}44.61 \pm \\
1.25\end{array}$ & $\begin{array}{c}272.75 \pm \\
5.36\end{array}$ & $\begin{array}{c}0.86 \pm \\
0.04\end{array}$ & $\begin{array}{c}7.16 \pm \\
0.26\end{array}$ & $\begin{array}{c}4.26 \pm \\
0.11\end{array}$ & $\begin{array}{c}2.86 \pm \\
0.16\end{array}$ \\
\hline $\begin{array}{l}\text { Female } \\
\text { AEEA }\end{array}$ & 200 & $\begin{array}{c}45.08 \pm \\
4.09^{\mathrm{a}}\end{array}$ & $\begin{array}{c}38.50 \pm \\
1.40^{\mathrm{a}}\end{array}$ & $\begin{array}{c}268.66 \pm \\
4.96^{\mathrm{a}}\end{array}$ & $\begin{array}{l}0.84 \pm \\
0.02^{\mathrm{a}}\end{array}$ & $\begin{array}{c}7.78 \pm \\
0.22^{\mathrm{a}}\end{array}$ & $\begin{array}{c}4.03 \pm \\
0.11^{\mathrm{a}}\end{array}$ & $\begin{array}{c}3.40 \pm \\
0.08^{\mathrm{a}}\end{array}$ \\
\hline $\begin{array}{l}\text { Female } \\
\text { AEEA }\end{array}$ & 400 & $\begin{array}{c}48.35 \pm \\
2.11^{\mathrm{a}}\end{array}$ & $\begin{array}{c}43.50 \pm \\
3.03^{\mathrm{a}}\end{array}$ & $\begin{array}{c}286.66 \pm \\
6.16^{\mathrm{a}}\end{array}$ & $\begin{array}{l}0.97 \pm \\
0.03^{\mathrm{a}}\end{array}$ & $\begin{array}{c}7.36 \pm \\
0.15^{\mathrm{a}}\end{array}$ & $\begin{array}{c}4.28 \pm \\
0.08^{\mathrm{a}}\end{array}$ & $\begin{array}{c}3.31 \pm \\
0.18^{\mathrm{a}}\end{array}$ \\
\hline
\end{tabular}

Data provided as mean $\pm \operatorname{SEM}(\mathrm{n}=6)$; ${ }^{\mathrm{a}} \mathrm{p}>0.05$ treated groups vs control.

Table 10: Assessment of kidney function test of mice in acute toxicity study

\begin{tabular}{cccc}
\hline Groups & $\begin{array}{c}\text { Dose } \mathbf{~ m g} / \mathbf{k g} \\
(\mathbf{P . O})\end{array}$ & $\begin{array}{c}\text { Urea } \\
(\mathbf{m g} / \mathbf{d l})\end{array}$ & $\begin{array}{c}\text { Uric acid } \\
(\mathbf{m g} / \mathbf{d l})\end{array}$ \\
\hline Control & $\begin{array}{c}\text { Distilled water } \\
(5 \mathrm{ml} /\end{array}$ & $26.90 \pm 2.81$ & $4.810 \pm 0.09$ \\
AEEA & 2000 & $28.38 \pm 1.33^{\mathrm{a}}$ & $4.616 \pm 0.07^{\mathrm{a}}$ \\
\hline
\end{tabular}

Data provided as mean $\pm \operatorname{SEM}(\mathrm{n}=6)$; ${ }^{\mathrm{a}} \mathrm{p}>0.05$ treated groups vs control.
Table 11: Assessment of kidney function test of rats in sub-acute toxicity study

\begin{tabular}{ccccc}
\hline Groups & $\begin{array}{c}\text { Dose } \mathbf{m g} / \\
\mathbf{k g}(\mathbf{p . o} .)\end{array}$ & Creatinine & Urea (mg/dl) & $\begin{array}{c}\text { Uric acid } \\
(\mathbf{m g} / \mathbf{d l})\end{array}$ \\
\hline $\begin{array}{c}\text { Control } \\
\text { male }\end{array}$ & $\begin{array}{c}\text { Distilled } \\
\text { water } \\
(5 \mathrm{ml} / \mathrm{kg})\end{array}$ & $0.7 \pm 0.05$ & $18.63 \pm 1.29$ & $2.3 \pm 0.15$ \\
& & & \\
Male AEEA & $200 \mathrm{mg} / \mathrm{kg}$ & $0.7 \pm 0.03 \mathrm{a}$ & $22.18 \pm 2.01 \mathrm{a}$ & $1.75 \pm 0.11 \mathrm{a}$ \\
Male AEEA & $400 \mathrm{mg} / \mathrm{kg}$ & $0.73 \pm 0.05 \mathrm{a}$ & $20.35 \pm 1.88 \mathrm{a}$ & $2.2 \pm 0.12 \mathrm{a}$ \\
Control & $\begin{array}{c}\text { Distilled } \\
\text { water }(5\end{array}$ & $0.8 \pm 0.02$ & $19.06 \pm 0.42 \mathrm{a}$ & $2.34 \pm 0.08$ \\
Female & $\mathrm{ml} / \mathrm{kg})$ & & & \\
& & & \\
Female & $200 \mathrm{mg} / \mathrm{kg}$ & $0.75 \pm 0.03 \mathrm{a}$ & $20.83 \pm 0.65 \mathrm{a}$ & $2.23 \pm 0.09 \mathrm{a}$ \\
AEEA & & & & \\
Female & $400 \mathrm{mg} / \mathrm{kg}$ & $0.8 \pm 0.02 \mathrm{a}$ & $20.73 \pm 1.29 \mathrm{a}$. & $2.38 \pm 0.14 \mathrm{a}$ \\
AEEA & & & & \\
\hline
\end{tabular}

Data provided as mean \pm SEM ( $\mathrm{n}=6)$; ap $>0.05$ treated groups vs control.

\section{Table 12: Assessment of relative organ weight ( $\mathrm{g}$ per $100 \mathrm{~g}$ body wt) in} acute toxicity study

\begin{tabular}{|c|c|c|c|c|c|c|c|}
\hline Groups & $\begin{array}{c}\text { Dose } \\
\text { |mg/kg } \\
\text { (p.o.) }\end{array}$ & $\begin{array}{l}\text { Heart } \\
\text { (g) }\end{array}$ & $\begin{array}{l}\text { Lungs } \\
\text { (g) }\end{array}$ & $\begin{array}{l}\text { Liver } \\
\text { (g) }\end{array}$ & $\begin{array}{l}\text { Spleen } \\
\text { (g) }\end{array}$ & $\begin{array}{l}\text { Left } \\
\text { kidney } \\
\text { (g) }\end{array}$ & $\begin{array}{c}\text { Right } \\
\text { kidney } \\
\text { (g) }\end{array}$ \\
\hline Control & $\begin{array}{c}\text { Distilled } \\
\text { water } \\
(5 \mathrm{ml} / \mathrm{kg})\end{array}$ & $\begin{array}{c}0.40 \pm \\
0.02\end{array}$ & $\begin{array}{c}1.01 \pm \\
0.06\end{array}$ & $\begin{array}{c}4.85 \pm \\
0.17\end{array}$ & $\begin{array}{c}0.50 \pm \\
0.02\end{array}$ & $\begin{array}{c}0.72 \pm \\
0.03\end{array}$ & $\begin{array}{c}0.65 \pm \\
0.02\end{array}$ \\
\hline AEEA & $\begin{array}{c}2000 \\
\mathrm{mg} / \mathrm{kg}\end{array}$ & $\begin{array}{l}0.74 \pm \\
0.03 \mathrm{a}\end{array}$ & $\begin{array}{l}1.05 \pm \\
0.10 \mathrm{a}\end{array}$ & $\begin{array}{l}5.76 \pm \\
0.69 \mathrm{a}\end{array}$ & $\begin{array}{l}0.49 \pm \\
0.04 \mathrm{a}\end{array}$ & $\begin{array}{l}0.77 \pm \\
0.08 \mathrm{a}\end{array}$ & $\begin{array}{l}0.70 \pm \\
0.07 \mathrm{a}\end{array}$ \\
\hline
\end{tabular}

Data provided as mean $\pm \operatorname{SEM}(\mathrm{n}=6) ;{ }^{\mathrm{a}} \mathrm{p}>0.05$ treated groups vs control.

Table 13: Assessment of relative organ weight (g per $100 \mathrm{~g}$ body wt) in sub-acute toxicity study

\begin{tabular}{|c|c|c|c|c|c|c|c|c|}
\hline Groups & Heart (g) & Lungs (g) & Liver (g) & Spleen (g) & Left kidney (g) & $\begin{array}{l}\text { Right kidney } \\
\text { (g) }\end{array}$ & Thymus (g) & $\begin{array}{c}\text { TESTES/ } \\
\text { OVARIES (g) }\end{array}$ \\
\hline Control Male & $0.352 \pm 0.006$ & $0.698 \pm 0.021$ & $2.658 \pm 0.041$ & $0.726 \pm 0.018$ & $0.473 \pm 0.013$ & $0.387 \pm 0.013$ & $0.293 \pm 0.015$ & $1.07 \pm 0.024$ \\
\hline $\begin{array}{l}\text { Male AEEA } \\
200 \mathrm{mg} / \mathrm{kg}\end{array}$ & $0.361 \pm 0.007^{a}$ & $0.762 \pm 0.044^{\mathrm{a}}$ & $2.515 \pm 0.059^{\mathrm{a}}$ & $0.758 \pm 0.013^{\mathrm{a}}$ & $0.459 \pm 0.020^{\mathrm{a}}$ & $0.375 \pm 0.012^{\mathrm{a}}$ & $0.257 \pm 0.018^{\mathrm{a}}$ & $1.07 \pm 0.050^{\mathrm{a}}$ \\
\hline $\begin{array}{c}\text { Male AEEA } 400 \\
\mathrm{mg} / \mathrm{kg}\end{array}$ & $0.362 \pm 0.009^{a}$ & $0.755 \pm 0.040^{a}$ & $2.726 \pm 0.069^{a}$ & $0.692 \pm 0.014^{\mathrm{a}}$ & $0.461 \pm 0.017^{\mathrm{a}}$ & $0.359 \pm 0.007^{\mathrm{a}}$ & $0.258 \pm 0.01^{\mathrm{a}}$ & $1.16 \pm 0.015^{\mathrm{a}}$ \\
\hline Control Female & $0.373 \pm 0.012$ & $0.756 \pm 0.023$ & $2.34 \pm 0.036$ & $0.628 \pm 0.035$ & $0.291 \pm 0.007$ & $0.367 \pm 0.12$ & $0.187 \pm 0.013$ & $0.499 \pm 0.044$ \\
\hline $\begin{array}{l}\text { Female AEEA } \\
200 \mathrm{mg} / \mathrm{kg}\end{array}$ & $0.391 \pm 0.015^{\mathrm{a}}$ & $0.742 \pm 0.037^{\mathrm{a}}$ & $2.463 \pm 0.074^{\mathrm{a}}$ & $0.762 \pm 0.075^{\mathrm{a}}$ & $0.295 \pm 0.019^{\mathrm{a}}$ & $0.342 \pm 0.005^{\mathrm{a}}$ & $0.197 \pm 0.008^{a}$ & $0.521 \pm 0.013^{a}$ \\
\hline $\begin{array}{l}\text { Female AEEA } \\
400 \mathrm{mg} / \mathrm{kg}\end{array}$ & $0.389 \pm 0.011^{\mathrm{a}}$ & $0.749 \pm 0.023^{\mathrm{a}}$ & $2.242 \pm 0.054^{\mathrm{a}}$ & $0.691 \pm 0.018^{a}$ & $0.276 \pm 0.010^{a}$ & $0.336 \pm 0.010^{a}$ & $0.204 \pm 0.007^{\mathrm{a}}$ & $0.513 \pm 0.021^{\mathrm{a}}$ \\
\hline
\end{tabular}

Data provided as mean \pm SEM $(\mathrm{n}=6)$; ${ }^{\mathrm{a}} \mathrm{p}>0.05$ treated groups vs control.

\section{Histopathological investigation Acute toxicity study in mice Lungs}

Normal alveoli with blood vessels and normal bronchioles were observed in histopathological investigation of lungs of control group of animals in both $10 \mathrm{x}$ and $40 \mathrm{x}$ magnifications. Normal alveoli with bronchioles was observed in treated group of animals in both the magnifications (Figure 6).

Liver

Histopathological section of liver in control group and AEEA treated group $(2000 \mathrm{mg} / \mathrm{kg})$ showed normal lobular architecture. The central

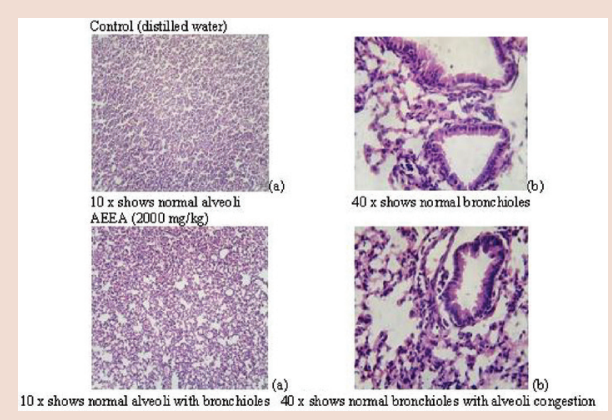

Figure 6: Histology of lungs from control and treated mice (10x (a) and 40x (b) magnifications) 
veins, sinusoids are found to be normal in the AEEA treated groups. Toxic signs like inflammation, fatty change or fibrosis were not found (Figure 7).

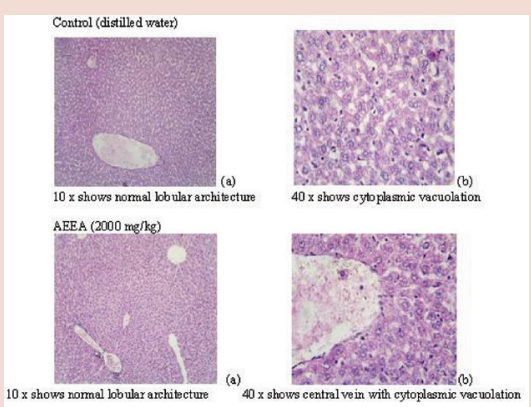

Figure 7: Histology of liver from control and treated mice groups (10x (a) and 40x (b)magnifications)

\section{Spleen}

Section from the spleen of control group and EAAE treated group (2000 $\mathrm{mg} / \mathrm{kg}$ ) showed red pulp congestion. No change was observed in white pulp, pencillar artery and the red pulp (Figure 8).

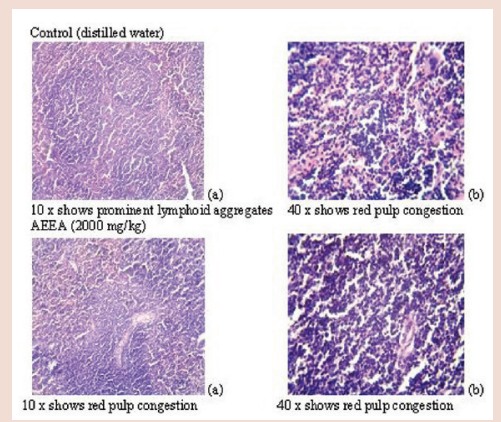

Figure 8: Histology of spleen from control and treated mice (10x (a) and 40x(b) magnifications)

\section{Kidney}

Sections from kidney of control group and AEEA treated group (2000 $\mathrm{mg} / \mathrm{kg}$ ) showed normal cortex, medulla and normal glomeruli. The interstitium was found to be normal and no inflammation or necrosis was observed (Figure 9).

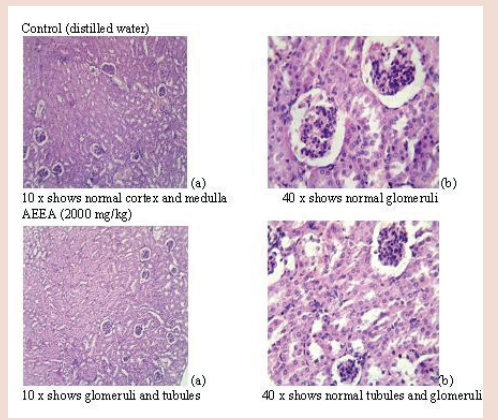

Figure 9: Histology of kidney from control and treated mice (10x (a) and 40x(b) magnifications)

\section{Sub-acute toxicity study in rats \\ Heart}

Section from the heart of control group and AEEA treated to male and female groups at a dose of $400 \mathrm{mg} / \mathrm{kg}$ showed normal myocytes (Figure 10 and 11).

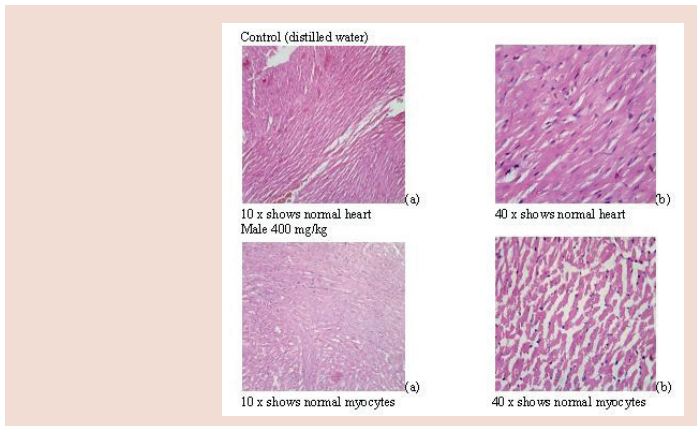

Figure 10: Histology of heart from control\& treated rat (male) (10x (a) and 40x (b) magnifications)

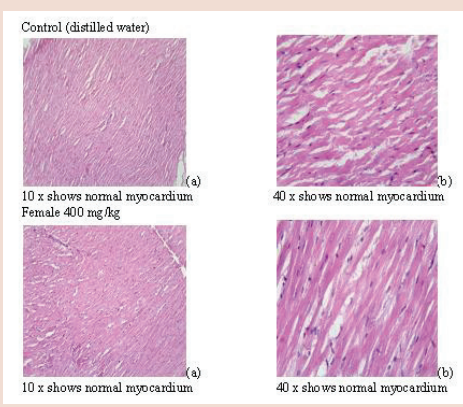

Figure 11: Histology of heart from control\& treated rats (female) (10x (a) \& $40 x$ (b) magnifications)

\section{Lungs}

Normal alveoli and normal bronchioles were observed in control group and AEEA treated to male and female groups at a dose of $400 \mathrm{mg} / \mathrm{kg}$ (Figure 12 and 13).

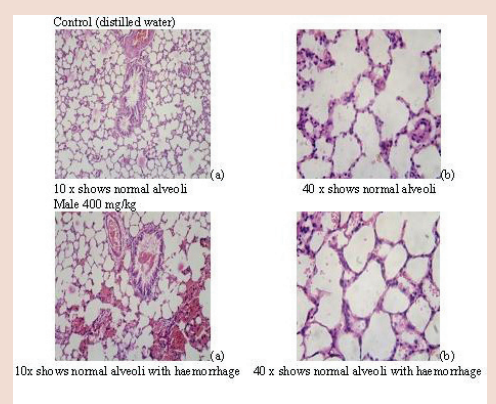

Figure 12: Histology of lungs from control\& treated rat (male) groups(10x (a)\&40x(b)magnifications)

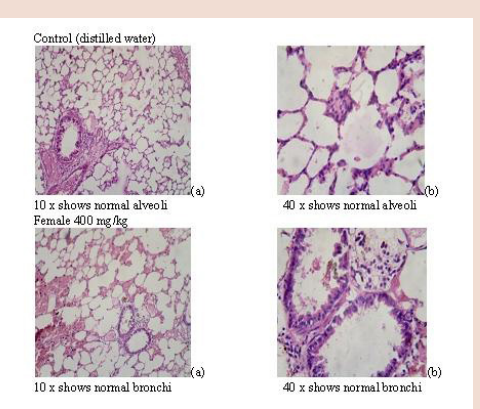

Figure 13: Histology of lungs from control\& treated (female) groups (10x(a)\&40x( b)magnifications) 


\section{Liver}

Histopathological section of liver in control group and AEEA treated to male and female groups at a dose of $400 \mathrm{mg} / \mathrm{kg}$ showed normal lobular architecture. The portal tracts, hepatocytes, central veins, sinusoids are found to be normal. No evidence of toxic signs observed as there is no inflammation, fatty change or fibrosis (Figure 14 and 15).

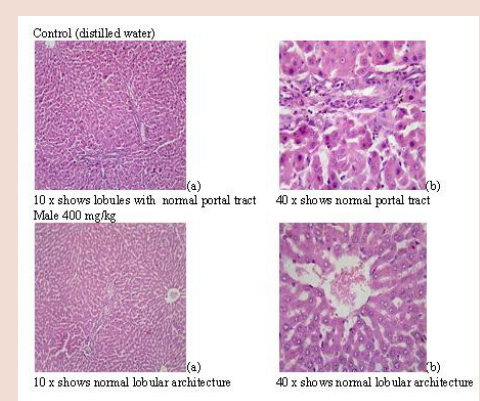

Figure 14: Histology of liver from control\& treated rat (male) groups(10x (a)\&40x(b) magnifications)

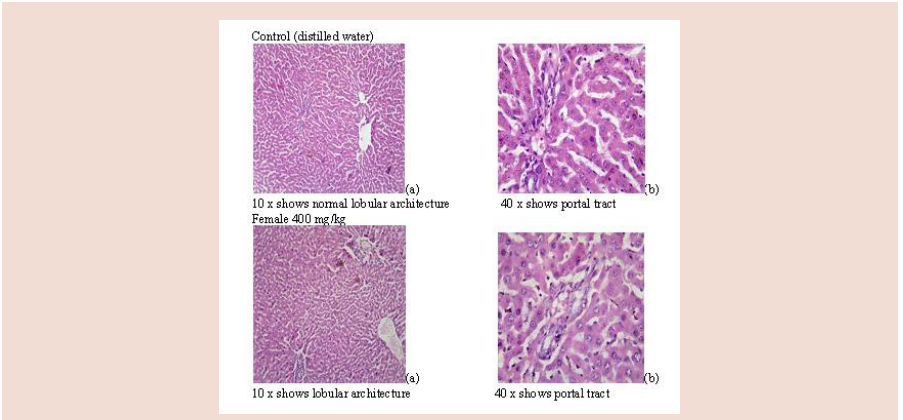

Figure 15: Histology of liver from control\& treated rats (female) (10x(a)\&40x(b) magnifications)

\section{Stomach}

Section from the stomach of control group and AEEA treated to male and female groups at a dose of $400 \mathrm{mg} / \mathrm{kg}$ showed normal mucosa, muscle layer and stomach glands (Figure 16 and 17).

\section{Spleen}

Section from the spleen of control group and AEEA treated to male and female groups at a dose of $400 \mathrm{mg} / \mathrm{kg}$ showed normal spleen with red

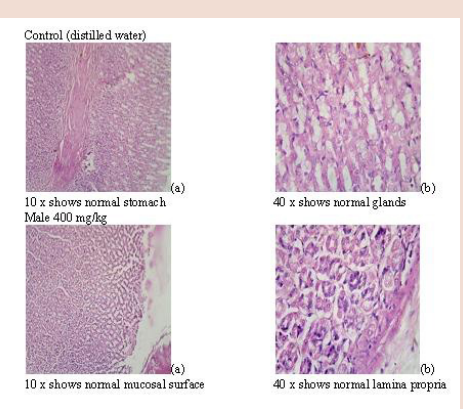

Figure 16: Histology of stomach from control\& treated rat (male) (10x(a) and 40x (b) magnifications)

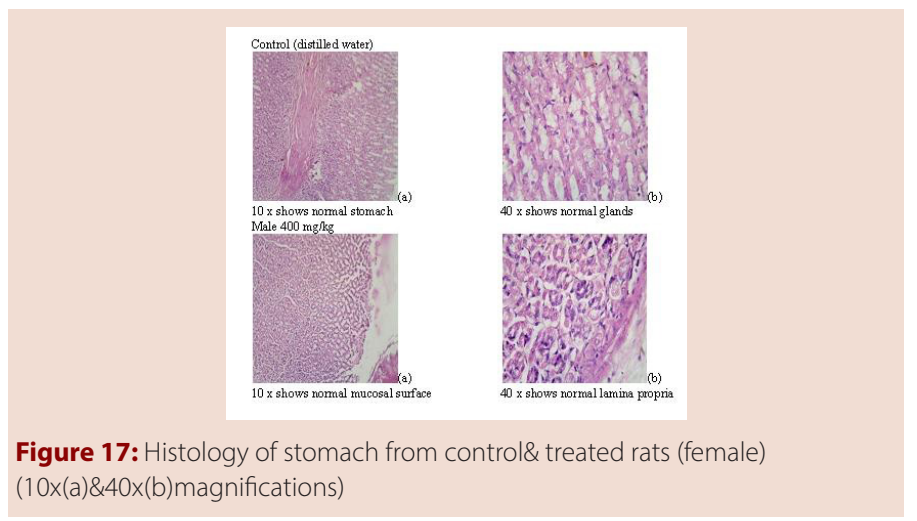

pulp and germinal center formation. No abnormality was observed in histological sections of spleen (Figure 18 and 19).
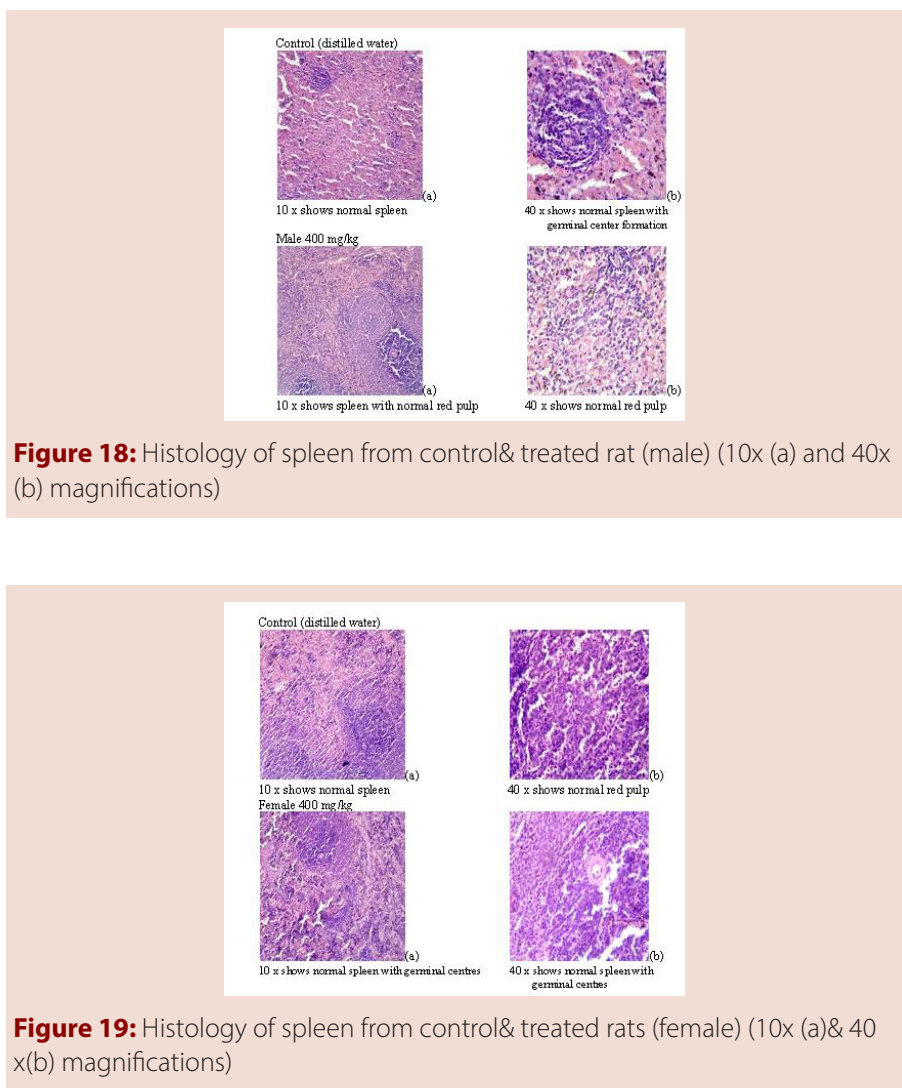

\section{Kidney}

Sections from kidney of control group and AEEA treated to male and female groups at a dose of $400 \mathrm{mg} / \mathrm{kg}$ showed normal cortex and medulla. The cortex showed normal glomeruli. The interstitium and distal convoluted tubules are found to be normal. No inflammation or tubular necrosis was observed (Figure 20 and 21).

\section{Testes}

Sections from kidney of control group and AEEA treated to male groups at a dose of $400 \mathrm{mg} / \mathrm{kg}$ showed normal testes and spermatozoa (Figure 22).

\section{Ovary}

Sections from kidney of control group and AEEA treated to female groups at a dose of $400 \mathrm{mg} / \mathrm{kg}$ showed normal ovarian follicles (Figure 23). 


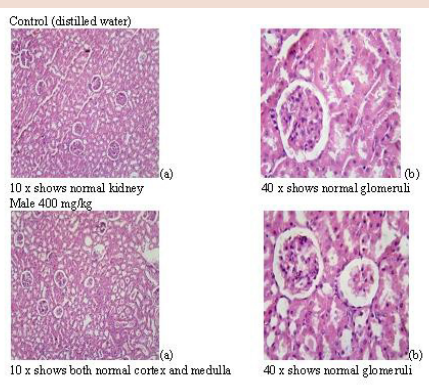

Figure 20: Histology of kidney from control\& treated rat (male) (10x (a) and 40x (b) magnifications)

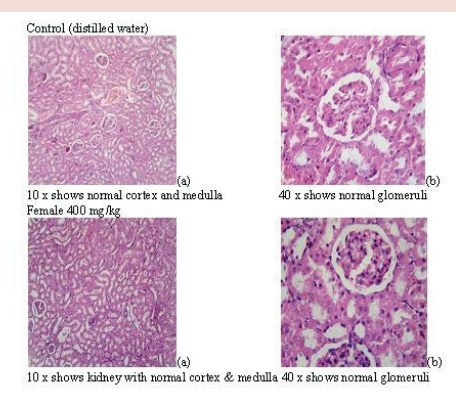

Figure 21: Histology of kidney from control\& treated rats (female) (10x (a)\& 40 $\mathrm{x}(\mathrm{b})$ magnifications)

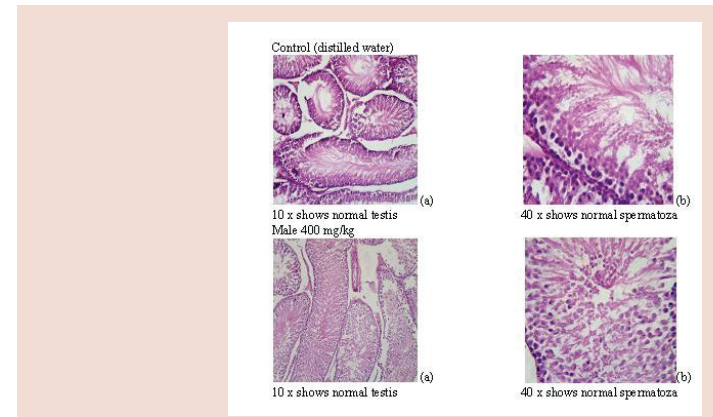

Figure 22: Histology of testes from control\& treated rat (male) (10x (a) and 40x (b) magnifications)

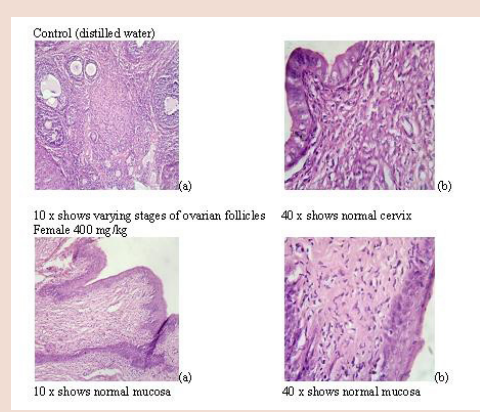

Figure 23: Histology of ovary from control\& treated rats (female) (10x (a) \& 40 $x(b)$ magnifications)

\section{DISCUSSION}

Acute and sub-acute studies in mice and rats were performed as per OECD guidelines 423 and 407 respectively to assess the safety profile of AEEA, as there were no earlier reports on the safety assessment of aqueous extract of Enicostemma axillare. In acute toxicity test, mortality was not observed in mice with the maximum dose of $2000 \mathrm{mg} / \mathrm{kg}$ throughout the 14 days of study. In sub-acute toxicity test also no mortality was observed in male and female rats with the maximum dose of 400 $\mathrm{mg} / \mathrm{kg}$ throughout 28 days of study. Significant changes were not found in breathing, sense of touch/sound, central nervous systems, behaviour pattern and locomotor activity. Convulsion, tremor, excessive salivation, diarrhea, sedation and edema were not observed.

Body weight and internal organ weight changes is sensitive and indicative marker for the first sign of toxicity when exposed to toxic substances. ${ }^{19}$ All animals were found to be active with increase in body weight both in acute and sub-acute toxicity study. Normal food and water consumption of animals clearly support the gain in body of animals throughout the study period.

Hematopoietic system is the most sensitive target for toxic substances and it is the important index of physiological and pathological status $\mathrm{s}^{20-21}$ and hence hematological investigation was carried out. No abnormality was observed in hematopoietic function indices for AEEA treated groups compared with control groups indicating the extract is safe.

Lipid profile assessment was performed for the animals treated with AEEA to verify any changes observed in serum levels of triglycerides, cholesterol and VLDL. ${ }^{22}$ No significant variation in cholesterol, triglycerides and VLDL levels in AEEA treated groups when compared with control groups.

SGOT, SGPT, ALP and bilirubin levels of the experimental animals were monitored as they are the specific markers for liver damage or injury. ${ }^{23}$ No significant changes in SGOT, SGPT, ALP and bilirubin level was observed in all the treated group animals. Thus animals treated with AEEA were found to be non-hepatotoxic.

To assess whether treatment of AEEA to rats causes any damage to kidney, kidney function test was performed. GLDH-UV Kinetic method, Jaffe method and Uricase POD method was adopted to evaluate the important markers of renal dysfunction viz. urea, uric acid and creatinine levels respectively. ${ }^{24}$ AEEA was found to be safe and nontoxic to kidney as there is no significant change observed in creatinine, urea and uric acid levels of AEEA treated group of animals both in acute and sub-acute toxicity when compared with control group animals. When compared with control groups, no variation in relative organ weight was observed for AEEA treated groups both in acute and sub-acute toxicity study.

The above mentioned biochemical investigations were in correlation with the histopathological studies.

Section of the heart of the control and AEEA treated animals showed no abnormality as shown in Figures 10 and 11. Alveoli and bronchioles were found to be normal both in control and treated group confirming that AEEA did not cause any toxicity to lungs of the animals. Inflammation or cirrhosis or necrosis was not observed in the histological study of liver in mice and rats tested with AEEA at a dose of $400 \mathrm{mg} / \mathrm{kg}$. Normal cortex, medulla and normal glomeruli were observed indicating that AEEA did not cause any damage to kidney. Cross section of stomach under low and high power magnification showed normal mucosal surface, normal stomach glands and normal lamina propria indicated that AEEA not caused any toxicity to rats. Histological sections of testes clearly implicated that no testicular toxicity was observed to the treated male group of rats for 28 days in sub-acute study. Observation of normal cervix, mucosa and follicles from histological sections of ovary confirmed that AEEA was non toxic to ovary of female rats. 


\section{CONCLUSIONS}

Based on our results, we conclude that AEEA were found to be safe up to a dose of $2000 \mathrm{mg} / \mathrm{kg} /$ day. Hematological, biochemical and histopathological investigations clearly demonstrates that single oral administration upto $2000 \mathrm{mg} / \mathrm{kg}$ in acute toxicity study and daily oral administration of the AEEA for 28 days upto $400 \mathrm{mg} / \mathrm{kg}$ in sub-acute toxicity study caused no damage to the organs like heart, lungs, liver, spleen and kidney. Abnormalities were also not observed in the organs stomach, testes and ovary of the animals tested at a dose of 200 and $400 \mathrm{mg} / \mathrm{kg}$ respectively. The study provided significant data on the sub-acute toxicity profile of AEEA which may be valuable in the clinical study of medicinal herb Enicostemma axillare. The aqueous extract of Enicostemma axillare thus may be used for manufacturing pharmaceutical formulations.

\section{ACKNOWLEDGEMENTS}

The authors thank All India Council for Technical Education, New Delhi whole heartedly for providing funds to carry out this research. The authors are also thankful to Kovai Medical Center Research and Educational Trust, Kovai Estate Kalapatti Road, Coimbatore, Tamilnadu for providing necessary facilities for carrying out the work.

\section{CONFLICT OF INTEREST}

The authors have declared that there is no Conflict of interest.

\section{ABBREVIATION USED}

EA: Enicostemma axillare (Lam.) Raynal; AEEA: Aqueous extract of Enicostemma axillare; OECD: Organisation for Economic Co-operation and Development PDAB: p-dimethyl aminobenzene; $\mathrm{RH}$ : Relative $\mathrm{Hu}$ midity; CPCSEA: Committee for the Purpose of Control and Supervision on Experiments on Animals; IAEC: Institutional Animal Ethical Committee; RBC : Red blood cells; WBC: White blood cells; Hb: Hemoglobin; PCV: Packed cell volume; MPV: Mean Platelet Volume; MCV: Mean corpuscular volume; $\mathrm{MCH}$ : Mean corpuscular hemoglobin; MCHC: Mean Corpuscular Hemoglobin Concentration; RDW: Red Blood Cell Distribution Width; VLDL: Very low density lipoprotein; SGOT : Serum glutamic oxaloacetic transaminase; SGPT: Serum glutamic-pyruvic transaminase; ALP: Alkaline phosphatase; GLDH: Glutamate dehydrogenase; POD: Peroxidase.

\section{REFERENCES}

1. Gamble JS. Flora of the Presidency of Madras. Rubiaceae to Euphorbiaceae; 1993. p. 870-83.

2. Vasu VT, Ashwinikumar C. Antidiabetic effect of Enicostemma littorale Blume aqueous extract in newly diagnosed non-insulin-dependent diabetes mellitus patients (NIDDM): a preliminary investigation. Orient Pharm Exp Med. 2003. 3(2): 84-9.
3. Singh VK, Govil JN, Gurdip S. Recent Progress in Medicinal Plants. Vol I Ethno medicine and Pharmacognosy. Sci Tech Publishing LLC, USA; 2002.

4. Babu PS, Prince PSM. Antihyperglycemic and antioxidant effects of hyponid, an Ayurvedic herbomineral formulation on streptozocin induced diabetic rats. $J$ Pharm Pharmacol. 2004; 56(11): 1435-42.

5. Murali B, Upadhyay UM, Goyal RK. Effect of chronic treatment with Enicostemma littorale in non-insulin-dependent diabetic (NIDDM) rats. $J$ Ethnopharmacol. 2002; 81(2): 199-204.

6. Viswakarma SL, Rakesh SD. Evaluation of effect of aqueous extract of Enicostemma littorale Blume in streptozotocin induced type 1 diabetes rats. Indian J Exp Biol. 2010; 48: 26-30.

7. Vijayvargia R, Kumar M, Gupta S. Diabetes mellitus in rats. Indian J Exp Biol. 2000; 38(8): 781-4.

8. Maroo J, Ghosh A. Antidiabetic efficacy of Enicostemma littorale methanol extract in alloxan-induced diabetic rats. Pharm Biol. 2003; 41(5): 388-91.

9. Srinivasan M, Padmanabhan M, Prince PSM. Effects of aqueous Enicostemma littorale blume extract on key carbohydrate metabolic enzymes, lipid peroxides and antioxidants in alloxan induced diabetic rats. J Pharm Pharmacol. 2005; 57(4): 497-503.

10. Niranj MB, Suparna B, Sarita G. Protective effect of Enicostemma littorale Blume on rat model of diabetic neuropathy. Am J Infect Dis. 2009; 5(2): 222-4.

11. Vishwakarma SL, Rajani M. A rapid method for the isolation of swertiamarin from Enicostemma littorale. Pharm Biol. 2004; 42(6): 400-3.

12. Jaishree $\mathrm{V}$, Badami S. Antinociceptive activity of swertiamarin isolated from Enicostemma axillare. Phytomed. 2009; 16(2): 227-32.

13. Senthilkumar KTM, Rajkapoor B, Kavimani S. Protective effect of Enicostemma littorale against CCl4-induced hepatic damage in rats. Pharm Biol. 2005; 43(5): 485-7.

14. Vasu VT, Modi H, Thaikoottathil JV, Gupta S. Hypolipidaemic and antioxidant effect of Enicostemma littorale Blume aqueous extract in cholesterol fed rats. J Ethnopharmacol. 2005; 101(1): 277-82.

15. Gopal R, Gnanamani A, Udayakumar R, Sadulla S. Enicostemma littorale Blume-A potential hypolipidemic plant. Nat Prod Rad. 2004; 3(6): 401-5.

16. Rajasekaran A, Arivukkarasu R, Murugesh S. Hepatoprotective effect of Adenema hyssopifolium G. Don (Gentianaceae) in carbon tetrachloride-induced hepatotoxicity in rats. Trop J Pharm Res. 2010; 9(2): 157-63.

17. Organisation for Economic Co-Operation and Development (OECD) Guidelines for testing of chemicals. Acute oral toxicity-acute toxic class method. \#423, Paris, France; 2001. 423

18. Organisation for Economic Co-Operation and Development (OECD), Guidelines for Testing of Chemicals. Repeated Dose 28-days Oral Toxicity Study in Rodents \#407, Paris, France; 1995.

19. Sireeratawong S, Lertprasertsuke N, Srisawat U, Thuppia A, Ngamjariyawat A, Suwanlikhid N, et al. Acute and sub-acute toxicity study of the distilled water extract from Tiliacora triandra (Colebr.) Diels in rats. Songklanakarin J Sci Tech. 2008; 30(5): 729-37.

20. Almanca CCJ, Saldanha SV, Sousa DR, Trivilin LO, Nunes LC, Porfírio LC, et al. Toxicological evaluation of acute and sub-chronic ingestion of hydroalcoholic extract of Solanum cernuum Vell. in mice. J Ethnopharmacol. 2011; 138(2): 508-12.

21. Mukinda JT, Eagkes FK. Acute and sub-acute oral toxicity profile of the aqueous extract of Polygala fruicosa in female mice and rats. J Ethnopharmacol. 2010; 128(1): 236-40.

22. Mansura A. Effect of Peristrophebi calyculata on lipid profile of P-407 induced hyperlipidemic Wistar rats. J Med Plants Res. 2011; 5(4): 490-4.

23. Thapa BR, Walia A. Liver function test and their interpretation. Indian J Paediatr. 2007; 74(7): 663-71.

24. Almdal TP, Vilstrup $\mathrm{H}$. Effect of streptozotocin induced diabetes and diet on nitrogen loss from organs and the capacity of urea synthesis in rats. Diabetologia 1987; 30(12): 952-6. 
PICTORIAL ABSTRACT

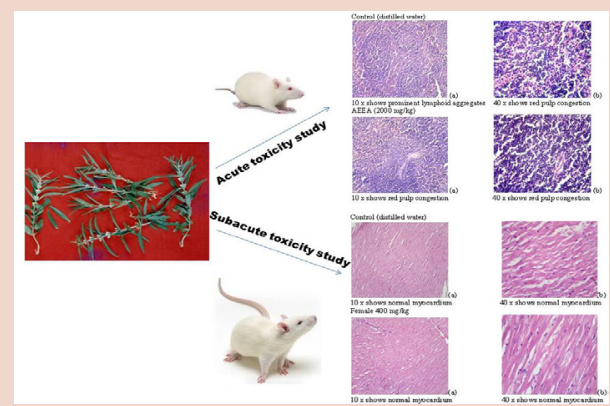

\section{SUMMARY}

- In acute oral toxicity study, the oral administration of AEEA in mice was found to be safe up to a dose of $2000 \mathrm{mg} / \mathrm{kg}$.

- Both male and female treated rats showed no change in hematological, biochemical and histological investigations.

- No signs of toxicity or mortality were observed in sub-acute toxicity study upto the dose of $400 \mathrm{mg} / \mathrm{kg}$ in rats.

\section{ABOUT AUTHORS}

Dr. A. Rajasekaran: Professor of KMCH College of Pharmacy is having more than 25 years of teaching and research experience in the field of pharmacy. He received research grants from Government agencies like DSIR, DST, DRDO and AICTE etc. He is having 5 patents to his credit. He has published 84 research papers in international and 110 research papers in National peer reviewed journals. He has visited Malaysia, Singapore, Thailand, Philippines, Sri Lanka and presented papers in various National and international conferences. He has received Best Teacher Award on 5 ${ }^{\text {th }}$ Sep 2010 from the Tamilnadu Dr. M.G.R medical University.

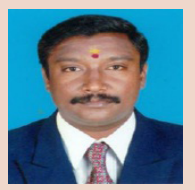

Dr. R. Arivukkarasu: Working as Assistant Professor in Department of Pharmacogonsy at KMCH College of Pharmacy has completed his PhD from SASTRA University, Thanjavur in 2013. He is having 15 years of teaching and 9 years of research experience in the field of pharmacognosy and phytochemistry. 